\title{
Cation coordination and hydrogen bonding in potassium and magnesium based-di-amidosil hybrids
}

\author{
S.C. Nunes ${ }^{a}$, V. de Zea Bermudez a,*, D. Ostrovskii ${ }^{\text {b }, ~ L . D . ~ C a r l o s ~}{ }^{\text {c }}$, \\ M.M. Silva ${ }^{\mathrm{d}}$, M.J. Smith ${ }^{\mathrm{d}}$ \\ a Departamento de Química and CQ-VR, Universidade de Trás-os-Montes e Alto Douro, 5000-911 Vila Real, Portugal \\ b Department of Applied Physics, Chalmers University of Technology, 41296 Göteborg, Swede \\ Departamento de Fisica and CICECO, Universidade de Aveiro, 3810-193 Aveiro, Portugal \\ Departamento de Química, Universidade do Minho, Gualtar, 4710-057 Braga, Portugal
}

Abstract

Fourier transform mid-infrared and Raman spectroscopies were employed to elucidate the cation/alkylene chains, cation/cross-link, cation/anion interactions and hydrogen bonding occurring in amorphous di-amide cross-linked alkylene/siloxane hybrid materials (diamidosils) doped with potassium and magnesium triflates $\left(\mathrm{KCF}_{3} \mathrm{SO}_{3}\right.$ and $\mathrm{Mg}\left(\mathrm{CF}_{3} \mathrm{SO}_{3}\right)_{2}$, respectively). Materials with compositions $\infty>n \geqslant 5$ (where $n$ expresses the molar ratio of carbonyl oxygen atoms per guest cation) were investigated. The conformations (gauche) of the alkylene chains of the host di-amidosil matrix are not affected by the presence of the guest salt in both doped di-amidosil families. The $\mathrm{K}^{+}$and $\mathrm{Mg}^{2+}$ ions coordinate to the carbonyl oxygen atoms of the amide cross-links within the whole rane of silt concentration const mat in the host matrix and, in the case of the $\mathrm{K}^{+}$-doped sample with $n=10$, to the formation of a new type of aggregate, stronger and more ordered than those detected at lower salt content. In both di-amidosil systems guest salt addition leads to the increase of ionic associated species and to a concomitant decrease of the concentration of "free" anions.

\section{Introduction}

Sol-gel chemistry $[1]$ is a remarkably versatile approach for the fabrication of organic-inorganic hybrid systems [2]. These advanced nanomaterials have attracted significant interest in the last few years due to the potential applications in many fields, such as, optics, magnetism and electrochemistry. The use of this synthetic route permits to combine at the nanosize level the properties of organic materials (e.g., elastomeric nature) with the features of inorganic silica frameworks (e.g., thermal and dimensional stability) in a single multifunctional nanocomposite. The experimental conditions of the Sol-gel process and the chemical nature of the organic and inorganic components (functionality of the silane precursor and concentration of reagents) dictate the morphology, nanostructure and degree of organization of the hybrids. The nature of the interactions established between the organic and inorganic components are also important. The latter aspect allows distinguishing two major subclasses in the class of hybrid materials, known as class I and class II, depending on the establishment or not of covalent bonds between the organic and inorganic components, respectively.

Recently, we introduced a novel family of class II hybrid materials, named di-amidosils. In these di-amide crosslinked alkylene/siloxane hybrid structures the alkylene chains are bonded at both ends to the siliceous framework through amide $(-\mathrm{NHC}(=\mathrm{O})-)$ groups [3]. The di-amidosil frameworks synthesized included 4 and 8 methylene repeat groups and were represented by the notations $\mathrm{d}-\mathrm{A}(4)$ and d-A(8), respectively, where d corresponds to di and A represents the amide cross-link. Both hybrid networks are room temperature white light emitters, presenting an emission large, broad band in the blue/purplish-blue spectral region. The origin of such band has been ascribed to the convolution of donor-acceptor pair (D-A) recombinations that occur in the NH groups of the amide linkages and in the siliceous nanodomains. The $\mathrm{d}-\mathrm{A}(8)$ di-amidosil has a quantum yield of $5.4 \%[3]$.

\footnotetext{
* Corresponding author. Tel.: +351 259350480; fax: +351 259350253.
} E-mail address: vbermude@utad.pt (V. de Zea Bermudez).
Aiming at producing materials for optical applications we incorporated very recently europium triflate $\left(\mathrm{Eu}\left(\mathrm{CF}_{3} \mathrm{SO}_{3}\right)_{3}\right)$ into the $\mathrm{d}-\mathrm{A}(8)$ di-amidosil host matrix [4]. Samples with $200 \leqslant n \leqslant 8$ were analyzed. The Fourier Transform infrared and Raman (FT-IR and FT-Raman, respectively) spectroscopic investigation performed provided evidences that: (1) The conformations of the alkylene chains of the host matrix in the doped materials (essentially gauche) are not affected by the presence of the salt; (2) The $\mathrm{Eu}^{3+}$ ions are coordinated to the $\mathrm{C}=\mathrm{O}$ groups of the amide cross-links over the whole range of salt concentration examined; (3) "Free" and weakly bonded triflate ions occur in all the samples analyzed and at $n=8$ an ionic aggregate of unknown nature is formed. Photoluminescence (PL) data demonstrated that the doped hybrids are room temperature white light emitters, due to the convolution of the hybrid host emitting centers (amide cross-linkages and siliceous nanodomains) and the $\mathrm{Eu}^{3+}$ intra- $4 \mathrm{f}^{6}$ ${ }^{5} \mathrm{D}_{0} \rightarrow{ }^{7} \mathrm{~F}_{0-4}$ transitions [4]. Another conclusion retrieved from $\mathrm{PL}$ results is that in the dilute di-amidosil with $n=100$ the $\mathrm{Eu}^{3+}$ ions occupy the same type of chemical local environment.

To confirm if, as we suspect, the cation coordination process in the di-amidosil hybrid medium depends on the nature of the guest cation, we have decided in the present work to extend the analysis of the doped d-A(8)based hybrids to the analogue series doped with $\mathrm{KCF}_{3} \mathrm{SO}_{3}$ and $\mathrm{Mg}\left(\mathrm{CF}_{3} \mathrm{SO}_{3}\right)_{2}$. With this goal, we have employed FT-IR and FT-Raman spectroscopies to determine if the presence of the guest salt affects the alkylene chain conformations and their state of disorder and to elucidate the cation/amide cross-link and cation/anion interactions, as well as hydrogen bonding. We have complemented this spectroscopic analysis with differential scanning calorimetry (DSC) and thermogravimetric analysis (TGA) data.

\section{Experimental section}

2.1. Materials

Ethanol $\left(\mathrm{CH}_{3} \mathrm{CH}_{2} \mathrm{OH}\right.$, Merck), pyridine (py, Aldrich) and tetrahydrofuran (THF, Merck) were stored over 
molecular sieves. Amberlyst A-21 Ion-Exchange Resin (Aldrich) was washed with THF and stored in an oven at $80^{\circ} \mathrm{C}$. Sebacoyl chloride $\left(\mathrm{ClC}(=\mathrm{O})\left(\mathrm{CH}_{2}\right)_{8} \mathrm{C}(=\mathrm{O}) \mathrm{Cl}\right.$, SC, Aldrich) and 3-aminopropyltriethoxysilane $\left(\left(\mathrm{CH}_{3} \mathrm{CH}_{2} \mathrm{O}\right)_{3}\right.$ $\mathrm{Si}\left(\mathrm{CH}_{2}\right)_{3} \mathrm{NH}_{2}$, APTES, Fluka), potassium triflate (Aldrich, $\mathrm{KCF}_{3} \mathrm{SO}_{3}$ ) and magnesium triflate (Aldrich, $\left.\mathrm{Mg}\left(\mathrm{CF}_{3} \mathrm{SO}_{3}\right)_{2}\right)$ were used as received. High purity distilled water $\left(\mathrm{H}_{2} \mathrm{O}\right)$ was used in all experiments.

\subsection{Synthesis}

The $\mathrm{K}^{+}$- and $\mathrm{Mg}^{2+}$-doped d-A(8)-based di-amidosils (Scheme 1) were prepared according to the method described in detail elsewhere [3,4]. Samples with $\infty>n \geqslant 5$ were produced as rigid films. Details of the synthetic procedure are indicated in the Table 1 .

The $\mathrm{K}^{+}$- and $\mathrm{Mg}^{2+}$-containing di-amidosils have been identified on the basis of the notation proposed previously [4], i.e., d- $\mathrm{A}(8)_{n} \mathrm{Y}\left(\mathrm{CF}_{3} \mathrm{SO}_{3}\right)_{x}$, where $\mathrm{Y}=\mathrm{K}(x=1)$ and $\mathrm{Mg}$ $(x=2)$.

\subsection{Experimental techniques}

Samples for TGA were transferred to open platinum crucibles and analyzed using a Rheometric Scientific TG 1000 thermobalance at a heating rate of $10^{\circ} \mathrm{min}^{-1}$ using high purity nitrogen as purging gas $\left(20 \mathrm{~cm}^{3} \mathrm{~min}^{-1}\right)$. Prior to measurement, the xerogels were vacuum-dried at $80^{\circ} \mathrm{C}$ for about $48 \mathrm{~h}$ and stored in an argon-filled glove box.

DSC measurements were obtained with a DSC131 Setaram DSC. Disk sections with masses of approximately $10-20 \mathrm{mg}$ were removed from the di-amidosil samples, placed in $40 \mu 1$ aluminium cans and stored in a desiccator over phosphorous pentoxide for one week at room temperature under vacuum. After this drying treatment the cans were hermetically sealed and the thermograms were recorded. Each sample was heated from 20 to $300^{\circ} \mathrm{C}$ at $10^{\circ} \mathrm{C} \mathrm{m^{-1 }}$. The purge gas used in all experiments was high purity nitrogen supplied at a constant $35 \mathrm{~cm}^{3} \mathrm{~min}^{-1}$ flow rate.

FT-IR spectra were acquired at room temperature using a Bruker 22 (Vektor) spectrometer placed inside a glovebox with a dry argon atmosphere. The spectra were col lected over the $4000-400 \mathrm{~cm}^{-1}$ range by averaging 150 scans at a spectral resolution of $2 \mathrm{~cm}^{-1}$. Solid samples $(2 \mathrm{mg})$ were finely ground, mixed with approximately $175 \mathrm{mg}$ of dried potassium bromide (Merck, spectroscopic grade) and pressed into pellets. Prior to recording the spectra, the pellets were first vacuum dried at $80-90^{\circ} \mathrm{C}$ for about $60 \mathrm{~h}$, to reduce the levels of adsorbed water and solvent, and then transferred into a glove-box.

The FT-Raman spectra were recorded at room temperature with a Bruker IFS-66 spectrometer equipped with a FRA-106 Raman module and a near-infrared YAG laser with wavelength of $1064 \mathrm{~nm}$. The spectra were collected over the $3200-300 \mathrm{~cm}^{-1}$ range at a resolution of $2 \mathrm{~cm}^{-1}$. The accumulation time for each spectrum was $4 \mathrm{~h}$.

To evaluate complex band FT-IR and FT-Raman envelopes and to identify underlying spectral components, the iterative least-squares curve-fitting procedure in the PeakFit ${ }^{1}$ software was used extensively throughout this study. The best fit of the experimental data was obtained by varying the frequency, bandwidth and intensity of the bands and by employing Voight band shapes. A linear baseline correction with a tolerance of $0.2 \%$ was employed. The standard errors of the curve-fitting procedure were less than 0.02 .

\section{Results and discussion}

\subsection{Morphology and thermal properties}

The DSC curves show that the d-A $(8)_{n} \mathrm{KCF}_{3} \mathrm{SO}_{3}$ and d-A $(8)_{n}\left(\mathrm{MgCF}_{3} \mathrm{SO}_{3}\right)_{2}$ xerogels with $n \geqslant 5$ are entirely amorphous (Fig. 1).

The TGA curves of selected $\mathrm{K}^{+}$-doped di-amidosils with $n \geqslant 5$ (Fig. 2) reveal that these materials start to decompose at about $280-290^{\circ} \mathrm{C}$. From approximately 340 $370^{\circ} \mathrm{C}$ the rate of mass loss increases dramatically (Fig. 2). In the salt-rich $\mathrm{K}^{+}$-based sample with $n=5$ degradation takes place in two stages: the first stage begins at $300^{\circ} \mathrm{C}$ and the second one is initiated at $485^{\circ} \mathrm{C}$ (Fig. 2).

In the case of the di-amidosils doped with $\mathrm{Mg}\left(\mathrm{CF}_{3} \mathrm{SO}_{3}\right)_{2}$ and $n \geqslant 5$, thermal decomposition starts at about 240 $270^{\circ} \mathrm{C}$ and proceeds with a progressive mass loss. Beyond $330-370^{\circ} \mathrm{C}$ the process is significantly more rapid (Fig. 2). At $n=10$ and 5, a second stage of degradation is visible in the TGA curves at temperatures higher than approximately $420^{\circ} \mathrm{C}$ (Fig. 2).

\subsection{Polymer chain conformations and state of disorder}

In this section we will examine the FT-Raman spectra of the d-A(8) ${ }_{n} \mathrm{KCF}_{3} \mathrm{SO}_{3}$ and d- $\mathrm{A}(8)_{n}\left(\mathrm{MgCF}_{3} \mathrm{SO}_{3}\right)_{2}$ di-amidosils in the region that includes the $\mathrm{CH}_{2}$ symmetric and asymmetric stretching modes $\left(\mathrm{v}_{\mathrm{s}} \mathrm{CH}_{2}\right.$ and $\mathrm{v}_{\mathrm{a}} \mathrm{CH}_{2}$, respectively). This high-frequency region $\left(3000-2800 \mathrm{~cm}^{-1}\right)$ is extremely useful to evaluate alterations of the polymer chain conformations and of their state of disorder in the presence of increasing amounts of guest salt.

The FT-Raman spectra of the d-A $(8)_{n} \mathrm{KCF}_{3} \mathrm{SO}_{3}$ and d$\Lambda(8)_{n} \mathrm{Mg}\left(\mathrm{CF}_{3} \mathrm{SO}_{3}\right)_{2}$ compounds in the $\mathrm{v}_{\mathrm{s}} \mathrm{CH}_{2}$ and $\mathrm{v}_{\mathrm{a}} \mathrm{CH}_{2}$ regions are represented in Figs. $3 a$ and $b$, respectively.

The frequency, intensity and frequency width at medium height of the Raman $v_{s} \mathrm{CH}_{2}$ and $v_{\mathrm{a}} \mathrm{CH}_{2}$ bands are sensitive to the gauche/trans conformer ratio, to order of the alkylene chains and to the intermolecular interactions occurring between the chains $[5,6]$. Shifts of these events to higher wavenumbers indicate an increase in the conformational disorder of the systems (i.e., an increase in the proportion of gauche conformations) [5,6]. In the Raman spectrum of alkylene chains in the crystalline state (all-trans conformations) the $\mathrm{v}_{\mathrm{a}} \mathrm{CH}_{2}$ mode is manifested as a strong band in the $2884-2878 \mathrm{~cm}^{-1}$ interval, whereas the $\mathrm{v}_{\mathrm{s}} \mathrm{CH}_{2}$ mode gives rise to bands at $2930 \mathrm{~cm}^{-1}$ (weak), $2900-2898 \mathrm{~cm}^{-1}$ (medium) and $2850-2844 \mathrm{~cm}^{-1}$ (strong) [7-9].

The FT-Raman $v \mathrm{CH}_{2}$ region of the $\mathrm{K}^{+} / \mathrm{Mg}^{2+}$-doped diamidosil hybrids with $100 \geqslant n \geqslant 5$ exhibit two prominent bands centered at 2924/2926 and 2892/2894 $\mathrm{cm}^{-1}$ and a shoulder at $2862 / 2863 \mathrm{~cm}^{-1}$ (Fig. 3a/Fig. 3b). The events at $2924 / 2926$ and $2862 / 2863 \mathrm{~cm}^{-1}$ are ascribed to the $v_{\mathrm{s}} \mathrm{CH}_{2}$ fundamental and to the Fermi resonance between the $v_{s} \mathrm{CH}_{2}$ fundamental with the many overtones of the $\mathrm{CH}_{2}$ bending vibrations, respectively, and are characteristic of disordered alkylene chains [7-9]. The intense 2892/ $2894 \mathrm{~cm}^{-1}$ feature is associated with the $\mathrm{v}_{\mathrm{a}} \mathrm{CH}_{2}$ stretching mode of alkylene chains in the amorphous state [7-9]. These results indicate that the alkylene chains of the diamidosil samples examined are fully disordered and adopt gauche conformations over the whole range of salt concentration considered. Thus, the spectroscopic data obtained

${ }^{1}$ Peakfit is a product of Jandel Corporation, 2591 Rerner Boulevard, San Rafael, CA 94901, USA. 
demonstrate that the addition of $\mathrm{KCF}_{3} \mathrm{SO}_{3}$ and $\mathrm{Mg}\left(\mathrm{CF}_{3} \mathrm{SO}_{3}\right)_{2}$ to d-A(8) affect, neither the conformations of the alkylene chains, nor their state of disorder.

3.3. Cation coordination and hydrogen bonding

In this section we will examine in detail the spectral signature of the $\mathrm{d}-\mathrm{A}(8)_{4} \mathrm{Y}\left(\mathrm{CF}_{3} \mathrm{SO}_{3}\right)_{x}$ di-amidosils as a function of salt content in specific regions of the FT-IR and FT-Raman spectra that permit to gain insight into the $\mathrm{Y}^{+x} /$ amide and $\mathrm{Y}^{+x} / \mathrm{CF}_{3} \mathrm{SO}_{3}^{-}$interactions.

3.3.1. Cationlamide interactions and hydrogen bonding

The assessment of the strength of the hydrogen bonds established between the amide cross-links and of the role of the amide linkages in the coordination of the $\mathrm{K}^{+}$and $\mathrm{Mg}^{2+}$ ions in the $\mathrm{d}-\mathrm{A}(8)$ system will be investigated here by means of a thorough analysis of the Amide I and Amide II regions $\left(1800-1600 \mathrm{~cm}^{-1}\right.$ and $1600-1500 \mathrm{~cm}^{-1}$ intervals, respectively) of the FT-IR spectra of the two series of diamidosils. As the di-amidosil host network provides a single type of coordinating site (the carbonyl oxygen atoms of amide groups), the analysis of the modifications undergone by the Amide I and Amide II bands upon salt addition are fundamental to determine to what extent the guest cation bond to the carbonyl oxygen atoms of the amide linkages in addition to interacting with the triflate oxygen atoms.

The FT-IR spectra of selected $\mathrm{K}^{+}$- and $\mathrm{Mg}^{2+}$-doped di-amidosil samples in the Amide I and Amide II spectral regions are depicted in Figs. $4 \mathrm{a}$ and $\mathrm{b}$, respectively. The results of the curve-fitting performed in the $1800-1500 \mathrm{~cm}^{-1}$ interval of the d-A(8) $\mathrm{KCF}_{3} \mathrm{SO}_{3}$ d-A $(8)_{n} \mathrm{Mg}\left(\mathrm{CF}_{3} \mathrm{SO}_{3}\right)_{2}$ di-amidosils are displayed in Fig. 5/ Fig. 6. The composition dependence of the area of the resolved components of the Amide I band of the two serie of samples is reproduced in the inset of Fig. 5/inset of Fig. 6 .

The Amide I mode (or carbonyl stretching mode) is a very complex vibration that receives a major contribution from the $\mathrm{C}=\mathrm{O}$ stretching vibration $[10]$. As this vibration mode is sensitive to the specificity and magnitude of hydrogen bonding, this band is often resolved into several distinct components, that correspond to different $\mathrm{C}=\mathrm{O}$ environments (aggregates). As the absorption coefficient of $\mathrm{C}=\mathrm{O}$ groups involved in different aggregates can be different and are unknown, it is not possible to compare intensity values of different spectral components. Therefore only the changes undergone by each component represent concentration variations of each type of aggregate [10,11]

The Amide II mode, essentially due to the $\mathrm{N}-\mathrm{H}$ inplane bending vibration, is sensitive to chain conformation and intermolecular hydrogen bonding [12], providing valuable information about the distribution of hydrogen bond strengths $[10,13]$.

Amide I region. Fig. 4 demonstrates that the addition of $\mathrm{K}^{+}$and $\mathrm{Mg}^{2+}$ ions to the d-A(8) matrix gives rise to several spectral modifications in the Amide I and Amide II regions, specially at high salt concentration, a solid proof that the alkali and alkali-earth ions coordinate to the carbonyl oxygen atoms of the amide cross-links within the whole range of salt concentration. We note that, while in the dilute $\mathrm{K}^{+}$- and $\mathrm{Mg}^{2+}$-doped compounds with $n \geqslant 20$ doping results in the disappearance of the $1754 \mathrm{~cm}^{-1}$ component and in a certain amount of band redistribution, in the case of more concentrated samples $(n<20)$ the spectral modifications are more marked (Figs. $4 a$ and b, respectively). This is particularly evident in the FT-IR spectrum of $\mathrm{d}-\mathrm{A}(8)_{10} \mathrm{KCF}_{3} \mathrm{SO}_{3}$, as manifested by the dramatic change suffered by the band profile (Fig. 4a).
The Amide I envelopes of the $\mathrm{K}^{+} / \mathrm{Mg}^{2+}$-doped diamidosils with $n \geqslant 20$ exhibit two broad bands centred at approximately $1733 / 1735$ and $1650 / 1648 \mathrm{~cm}^{-1}$, respectively (Fig. 4a/Fig. 4b). The $1735 \mathrm{~cm}^{-1}$ event was decomposed into two components at 1735 and $1714 / 1710 \mathrm{~cm}^{-1}$ (Fig. 5/Fig. 6), ascribed to disordered amide-amide aggregates (D1 and D2, respectively) in which the hydrogen bonds between the $\mathrm{N}-\mathrm{H}$ and $\mathrm{C}=\mathrm{O}$ groups are very weak and weak, respectively [3]. The broad band at 1650/ $1648 \mathrm{~cm}^{-1}$ produced by the $\mathrm{K}^{+} / \mathrm{Mg}^{2+}$-doped $\mathrm{d}-\mathrm{A}(8)$-based compounds with $n \geqslant 20$ was resolved into three components at $1679 / 1675,1652 / 1648$ and $1623 / 1618 \mathrm{~cm}^{-1}$ (Fig. 5/Fig. 6). These features are attributed to the absorption of $\mathrm{C}=\mathrm{O}$ groups in ordered amide-amide aggregates with increased hydrogen bond strength $(\mathrm{O} 1, \mathrm{O} 2$ and $\mathrm{O} 3$, respectively) [3].

The new Amide I event observed at $1605 \mathrm{~cm}^{-1}$ in FT-IR spectrum of $\mathrm{d}-\mathrm{A}(8) \mathrm{KCF}_{3} \mathrm{SO}_{3}$ (Fig. 5) is indicative of the occurrence of a new ordered amide-amide aggregate, considerably stronger that those found in the more diluted samples. In the Amide I envelope of the d-A $(8)_{n} \mathrm{Mg}\left(\mathrm{CF}_{3} \mathrm{SO}_{3}\right)_{2}$ materials with $n<10$ band redistribution is more marked than that observed in the less concentrated samples (Fig. 6).

The results retrieved from the Amide I region of the $\mathrm{K}^{+}$/ $\mathrm{Mg}^{2+}$-doped xerogels with $n<20$ may be correlated with a massive coordination of the cations to the carbonyl groups of the amide linkages of the hydrogen-bonded amideamide aggregates of $\mathrm{d}-\mathrm{A}(8)$ [3]. While for $\mathrm{K}^{+}$doping this process involves a considerable breakdown of the ordered $\mathrm{O} 2$ structures (Fig. 5), in the presence of $\mathrm{Mg}^{2+}$ ions the main consequence is a remarkable increase of the proportion of aggregates $\mathrm{O} 3$ (Fig. 6).

The plots shown in the inset of Fig. 5/inset of Fig. 6 allow to gain better insight into the modifications the hydrogen-bonded aggregates of $\mathrm{d}-\mathrm{A}(8)$ are subject to as the $\mathrm{KCF}_{3} \mathrm{SO}_{3} / \mathrm{Mg}\left(\mathrm{CF}_{3} \mathrm{SO}_{3}\right)_{2}$ concentration is progressively raised.

The component characteristic of non-bonded $\mathrm{C}=\mathrm{O}$ groups $\left(1754 \mathrm{~cm}^{-1}\right)$ in the $\mathrm{K}^{+}$-doped $\mathrm{d}$-A $(8)$-based series disappears upon salt addition $(n=80)$, the amount of disordered D1 amide-amide aggregates suffers a slight increase, whereas the proportion of the disordered D2 and ordered $\mathrm{OI}$ ones remains practically unchanged. In addition, the fraction of the most ordered $\mathrm{O} 3$ associations decreases slightly, whereas that of the ordered $\mathrm{O} 2$ ones increases rapidly (inset of Fig. 5). Between $80>n \geqslant 40$ the only changes worth mentioning are a marked decrease of the proportion of the disordered D1 aggregates at the expense of a slight increase of the amount of $\mathrm{D} 2$ and $\mathrm{O} 2$ associations (inset of Fig. 5)

In the $\mathrm{Mg}^{2+}$-doped di-amidosils with $n \geqslant 20$, while the fraction of non-bonded $C=O$ groups $\left(1754 \mathrm{~cm}^{-1}\right)$ is null, the proportion of the disordered D1 and D2 and ordered O1 aggregates remains practically constant (inset of Fig. 6). In contrast, the proportion of ordered $\mathrm{O} 2$ and O3 structures suffers an increase at $n=100$ (inset of Fig. 6). Upon inclusion of more salt $(n<100)$, the fraction of $\mathrm{O} 2$ structures grows significantly and in parallel that of the $\mathrm{O} 3$ aggregates decreases (inset of Fig. 6). At $n=5$ the most significant change is, no doubt, the increase of the amount of $\mathrm{O} 3$ associations (inset of Fig. 6).

Amide II. The Amide II band of the $\mathrm{d}-\mathrm{A}(8)_{n} \mathrm{KCF}_{3} \mathrm{SO}_{3}$ and $\mathrm{d}-\mathrm{A}(8)_{n} \mathrm{Mg}\left(\mathrm{CF}_{3} \mathrm{SO}_{3}\right)_{2}$ di-amidosils with $n \geqslant 20$ was decomposed into a pair of peaks at 1562/1557 and 1532/ $1538 \mathrm{~cm}^{-1}$, respectively (Fig. 5/Fig. 6), suggesting the presence of hydrogen-bonded aggregates with two average hydrogen bond strengths. Although these bands persist in 
the FT-IR spectrum of the $\mathrm{d}-\mathrm{A}(8){ }_{10} \mathrm{KCF}_{3} \mathrm{SO}_{3}$ di-amidosil, a new component emerges at $1585 \mathrm{~cm}^{-1}$ (Fig. 5). This feature is tentatively correlated with the development of the Amide $\mathrm{I}$ band at $1605 \mathrm{~cm}^{-1}$ (Fig. 5). We note that both components (i.e., 1605 and $1585 \mathrm{~cm}^{-1}$ ) are partly superimposed in the borderline that is usually established to distinguish the Amide I region from the Amide II region.

\subsection{Cation/anion interaction}

As mentioned above, in the two sets of doped di-amidosils studied in the present work, the triflate oxygen atoms are, apart from the oxygen atoms of the carbonyl groups of the amide cross-links, the second type of coordinating site available for the guest $\mathrm{K}^{+}$and $\mathrm{Mg}^{2+}$ ions.

To inspect the chemical surrounding experienced by the triflate ions at increasing salt content in both series of materials we analysed the FT-IR and FT-Raman spectra in the diagnostic region characteristic of the symmetric stretching vibration mode of the $\mathrm{SO}_{3}$ group $\left(v_{5} \mathrm{SO}_{3}\right)$, since this vibration mode is very sensitive to coordination effects.

The components isolated in the FT-IR/FT-Raman $v_{\mathrm{s}} \mathrm{SO}_{3}$ band profiles of representative $\mathrm{K}^{+}$and $\mathrm{Mg}^{2+}$-doped di-amidosils are depicted in Figs. $7 \mathrm{a}$ and b, respectively. Figs. $8 \mathrm{a}$ and $\mathrm{b}$ show the composition dependence of the area of the resolved components of the d$\mathrm{A}(8)_{n} \mathrm{KCF}_{3} \mathrm{SO}_{3}$ and $\mathrm{d}-\mathrm{A}(8)_{n} \mathrm{Mg}\left(\mathrm{CF}_{3} \mathrm{SO}_{3}\right)_{2}$ xerogels with $n \leqslant 20$, in the FT-IR and FT-Raman $v_{\mathrm{s}} \mathrm{SO}_{3}$ envelopes, respectively.

The FT-IR/FT-Raman $v_{\mathrm{s}} \mathrm{SO}_{3}$ band of the $\mathrm{K}^{+}$-doped dA(8)-based samples with $n \geqslant 10 / 20$ was decomposed into three components: one intense event at $1032 \mathrm{~cm}^{-1}$ and two shoulders at 1038 and $1028 \mathrm{~cm}^{-1}$ (top/bottom of Fig. 7a). The FT-IR/FT-Raman $v_{5} \mathrm{SO}_{3}$ regions of the salt-rich ormolyte with $n=5$ (top/bottom of Fig. 7a) were decomposed into the same components: three bands at 1052,1038 and $1032 \mathrm{~cm}^{-1}$ and two shoulders at 1045 and $1028 \mathrm{~cm}^{-1}$.

In contrast, the FT-IR $v_{5} \mathrm{SO}_{3}$ envelope of the d$\mathrm{A}(8)_{n} \mathrm{Mg}\left(\mathrm{CF}_{3} \mathrm{SO}_{3}\right)_{2}$ samples with $n \geqslant 20$ was resolved into only two components: a band at $1032 \mathrm{~cm}^{-1}$ and a shoulder at $1028 \mathrm{~cm}^{-1}$ (top of Fig. 7b). Upon introduction of more guest salt $(n=5)$ the FT-IR $v_{\mathrm{s}} \mathrm{SO}_{3}$ band profile changes drastically as a result of the growth of new events at 1051,1045 and $1038 \mathrm{~cm}^{-1}$ (top of Fig. $7 \mathrm{~b}$ ). In the case of d- $\mathrm{A}(8)_{5} \mathrm{Mg}\left(\mathrm{CF}_{3} \mathrm{SO}_{3}\right)_{2}$ a new component develops at $1051 \mathrm{~cm}^{-1}$ in the FT-IR spectra (top of Fig. 7b). The FT-Raman $v_{s} \mathrm{SO}_{3}$ envelope of the d- $\mathrm{A}(8)_{n} \mathrm{Mg}\left(\mathrm{CF}_{3} \mathrm{SO}_{3}\right)_{2}$ samples with $n \geqslant 20$ was decomposed into a band at $1032 \mathrm{~cm}^{-1}$ and two shoulders at 1037 and $1026 \mathrm{~cm}^{-1}$ (bottom of Fig. 7b). At $n=10$ a weak band emerges at approximately $1009 \mathrm{~cm}^{-1}$ in the FT-Raman spectrum (bottom of Fig. 7b). With the increase of salt concentration $(n=5)$ the intensity of the shoulders detected in the dilute materials increases and a new band appears at $1043 \mathrm{~cm}^{-1}$ (bottom of Fig. 7b).

The $v_{\mathrm{s}} \mathrm{SO}_{3}$ band at $1032 \mathrm{~cm}^{-1}$ is assigned to "free" ions [14]. The contribution of "cross-link separated ion pairs" to the $1032 \mathrm{~cm}^{-1}$ band cannot be, however, discarded [15]. The shoulders found at $1028 / 1026 \mathrm{~cm}^{-1}$ and at $1038 / 1037 \mathrm{~cm}^{-1}$ are associated with weakly coordinated triflate ions [15]. The event at about $1045 / 1043 \mathrm{~cm}^{-1}$ is tentatively ascribed to contact ion-pairs. The 1052/1051 and $1009 \mathrm{~cm}^{-1}$ features are tentatively attributed to the formation of ionic aggregates of unknown nature.
Close analysis of the FT-IR and FT-Raman spectra of the $\mathrm{d}-\mathrm{A}(8)_{n} \mathrm{KCF}_{3} \mathrm{SO}_{3}$ samples (top and bottom of Fig. 8a, respectively) allows to infer that the vibration of the five $v_{\mathrm{s}} \mathrm{SO}_{3}$ modes present is manifested differently in infrared and in Raman scattering. This means that the vibration of each of these modes imposes changes of different magnitude in the dipole moment and in the polarizability of the species in question. This effect is particularly evident in the case of the FT-IR components located at 1052 and $1045 \mathrm{~cm}^{-1}$, whose counterparts in the FT-Raman spectrum are much weaker. In the FT-IR spectra of the $\mathrm{Mg}^{2+}$-based analogues, the component at $1051 \mathrm{~cm}^{-1}$ $(n=5)$ (top of Fig. 8b) has no counterpart in the FTRaman spectrum (bottom of Fig. $8 \mathrm{~b}$ ).

The presence of $\mathrm{KCF}_{3} \mathrm{SO}_{3}\left(\mathrm{Mg}\left(\mathrm{CF}_{3} \mathrm{SO}_{3}\right)_{2}\right)$ in the d-A(8) di-amidosil hybrid medium has the following major consequences:

(1) At $n \geqslant 20$, all the amide cross-links are saturated, but the $\mathrm{K}^{+}$(or $\mathrm{Mg}^{2+}$ ) ions disturb the amide-amide aggregates moderately. We have found evidence of a certain amount of aggregate redistribution, but globally the strength of hydrogen bonding remains the same, as manifested in Fig. 5 (Fig. 6).

(2) At $n<20$ the interaction of the $\mathrm{K}^{+}$ions with the $\mathrm{C}=\mathrm{O}$ groups is such that one amide-amide aggregate, stronger than those formed in the non-doped host matrix, develops. Fig. 8a (Fig. 8b) demonstrates that at $n \leqslant 10$, ionic association increases abruptly at the expense of a marked reduction of the concentration of "free" triflate ions.

Comparison of the spectral data obtained in the present work with the results derived from our previous study of the d-A(8) ${ }_{n} \mathrm{Eu}\left(\mathrm{CF}_{3} \mathrm{SO}_{3}\right)_{3}$ di-amidosils [4] leads us to conclude that the amide-amide agoregates of the salt-free network are more perturbed by the inclusion of $\mathrm{Eu}\left(\mathrm{CF}_{3} \mathrm{SO}_{3}\right)_{3}$ than by the incorporation of $\mathrm{KCF}_{3} \mathrm{SO}_{3}$ or $\mathrm{Mg}\left(\mathrm{CF}_{3} \mathrm{SO}_{3}\right)_{2}$ In fact, the growth of a new hydrogen-bonded aggregate appears much earlier in the presence of the guest lanthanide ion than in the presence of the $\mathrm{K}^{+}$ion $(n=80[4]$ and 10 , respectively). With these ions, the cation/amide interaction leads to the formation of the same amideamide association, an indication that hydrogen bonding is of the same order of magnitude and consequently independent of cation nature and concentration. In contrast, when $\mathrm{Mg}^{2+}$ ions are added to d-A(8), no new hydrogenbonded associations grow. These findings give support to the explanation that in the $\mathrm{d}-\mathrm{A}(8)$ medium the $\mathrm{Eu}^{3+}$ ions are the species that exhibit the greatest tendency to bond to the carbonyl oxygen atoms of the amide cross-links, followed by the $\mathrm{K}^{+}$ions. The $\mathrm{Mg}^{2+}$ ions have clearly less affinity for the $\mathrm{C}=\mathrm{O}$ groups than $\mathrm{Eu}^{3+}$ and $\mathrm{K}^{+}$.

Taking into account the latter conclusions, we would expect to find a very low proportion of coordinated triflate ions within the $\mathrm{d}-\mathrm{A}(8)_{n} \mathrm{Eu}\left(\mathrm{CF}_{3} \mathrm{SO}_{3}\right)_{3}$ medium. The FT-IR [16] and FT-Raman spectra of the $\mathrm{Eu}^{3+}$-doped di-amidosils confirmed the presence of a remarkably low degree of ionic association in the materials, even in salt-rich samples [4].

\section{Conclusions}

In the present work, we investigated amorphous diamidosils doped with a wide range of $\mathrm{KCF}_{3} \mathrm{SO}_{3}$ and $\mathrm{Mg}\left(\mathrm{CF}_{3} \mathrm{SO}_{3}\right)_{2}$ concentration $(\infty \geqslant n \geqslant .5)$. The studies carried out provided evidences that the alkylene chains in 
both doped di-amidosil series adopt essentially gauche conformations, demonstrating that the polymer chains of the host di-amidosil matrix are not affected by the presence of the guest salt at any salt content. The cations bond to the carbonyl oxygen atoms of the amide cross-links within the whole range of salt content. The concentration of "free" $\mathrm{CF}_{3} \mathrm{SO}_{3}^{-}$ions decreases progressively with the incorporation of guest salt in both systems, while those of the weakly coordinated triflate ions, contact ions pairs and higher aggregates grows.

\section{Acknowledgement}

This work was supported by Fundação para a Ciência e Tecnologia (SFRH/BD/13559/03 and POCTI/P/CTM/ 46780/03).

\section{References}

[1] C.J. Brinker, G. Scherer, Sol-gel Science: The Physics and Chemistry of Sol-gel Processing, Academic Press, San Diego, CA, 1990.

2] P. Gomez-Romero, C. Sanchez (Eds.), Functional Hybrid Materials, Wiley Interscience, New York, 2003.

[3] S.C. Nunes, V. de Zea Bermudez, J. Cybinska, R.A. Sá Ferreira, J. Legendziewicz, L.D. Carlos, M.M. Silva, M.J. Smith, D. Ostrovskii, Legendziewicz, L.D. Carlos, M.M. Silva, M.J. S
J. Rocha, J. Mater. Chem. 15 (2005) 3876-3886.

[4] S.C. Nunes, V. de Zea Bermudez, Y. Cybinska, R.A. Sá Ferreira, L.D C.C. Nunes, V. de Zea Bermudez, J. Cybinska, R.A. Sá Ferreira, L.D.
Carlos, Legendziewicz, M.M. Silva, M.J. Smith, D. Ostrovskii, Carlos, J. Legendziewicz, M.M. Silva, M.J. Smith, D. Ostrovskii,
Structure and photoluminescence of di-amidosil nanohybrids incorporating europium triflate, J. Alloy. Compd., submitted for porating curopium triflet, J. Klloy. Compd., sthanited for publication.

] R.A. Vaia, R.K. Teukolsky, E.P. Gianneli, Chem. Mater. 6 (1994) 1017-1022.

6] R.G. Snyder, H.L. Strauss, C.A. Ellinger, J. Phys. Chem. 86 (1982) $5145-5150$.

7] R. Wang, G. Baran, S.L. Wunder, Langmuir 16 (15) (2000) 6298 6305 .

8] N.V. Venkataraman, S. Vasudevan, J. Phys. Chem. B 105 (32) (2001) $7639-7650$.

9] K.G. Brown, E. Bicknell-Brown, M. Ladjadj, J. Phys. Chem. 91 (1987) 3436-3442.

10] D.J. Skrovanek, S.E. Howe, P.C. Painter, M.M. Coleman, Macromolecules 18 (1985) 1676-1683.

11] M.M. Coleman, K.H. Lee, D. Skrovanek, P.C. Painter, Macromolecules 19 (1986) 2149-2157.

12] T.M. Miyazawa, T. Shimanouchi, S.I. Mizushima, J. Chem. Phys. 24 (2) (1956) $408-418$.

(13) D.J.S. Skrovanek, P.C. Painter, M.M. Coleman, Macromolecules 19 (1986) 699-705.

14] $\AA$ Wendsjō, J. Lindgren, J.O. Thomas, G.C. Farrington, Solid State Ionics 53-56 (1992) 1077-1082.

[15] V. de Zea Bermudez, D. Ostrovskii, S. Lavoryk, M.C. Gonçalves, L.D. Carlos, Phys. Chem. Chem. Phys. 6 (3) (2004) 649-658.

[16] S.C. Nunes, V. de Zea Bermudez, D. Ostrovskii, unpublished work 


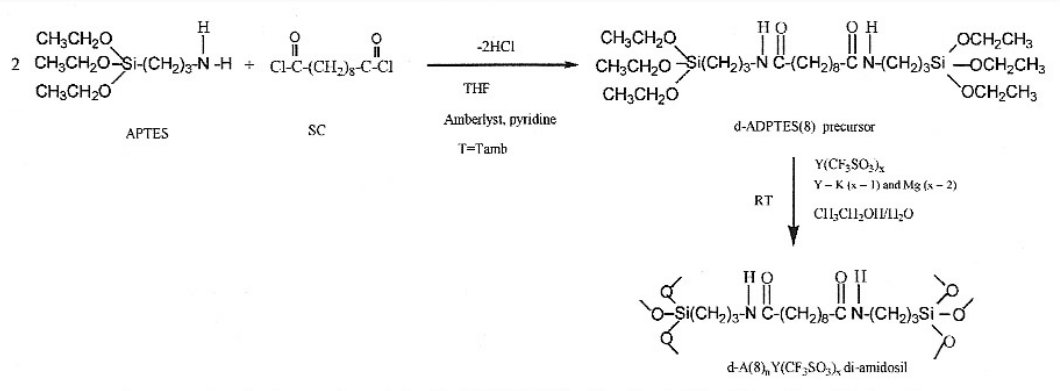

Scheme 1. Synthetic procedure of the $\mathrm{d}-\mathrm{A}(8)_{n} \mathrm{Y}\left(\mathrm{CF}_{3} \mathrm{SO}_{3}\right)_{x}$ di-amidosils $(\mathrm{Y}=\mathrm{K}(x=1)$ or $\mathrm{Mg}(x=2))$.

Table 1

Details of the synthetic procedure of the $\mathrm{d}-\mathrm{A}(8)_{n} \mathrm{KCF}_{3} \mathrm{SO}_{3}$ and d-A $(8)_{n} \mathrm{Mg}\left(\mathrm{CF}_{3} \mathrm{SO}_{3}\right)_{2}$ di-amidosils d- $\mathrm{A}(8)_{\mathrm{Y}} \mathrm{Y}\left(\mathrm{CF}_{3} \mathrm{SO}_{3}\right)_{x}$

$\begin{array}{lll} & & \\ \text { Fist step } & 0.5 \mathrm{~mL} & 2.3 \mathrm{mmol} \\ \text { SC } & 0.769 \mathrm{~mL} & 4.7 \mathrm{mmol} \\ \text { APTES } & 0.84 \mathrm{~g} & 2.8 \mathrm{mmol} \\ \text { Amberlyst resin } & 0.076 \mathrm{~mL} & 0.47 \mathrm{mmol} \\ \text { Py } & 20 \mathrm{~mL} & \\ \text { THF } & & \\ \text { Second step } & 1.096 \mathrm{~mL} & 18.7 \mathrm{mmol} \\ \mathrm{CH}_{3} \mathrm{CH}_{2} \mathrm{OH} & 0.169 \mathrm{~mL} & 9.4 \mathrm{mmol} \\ \mathrm{H}_{2} \mathrm{O} & \\ \text { THF } & 10 \mathrm{~mL} & \end{array}$

$n \quad \frac{\mathrm{d}-\mathrm{A}_{(}(8)_{n} \mathrm{KCF}_{3} \mathrm{SO}_{3}}{\left(\mathrm{~K}+\mathrm{C}^{2}\right.}$ $\mathrm{d}-\mathrm{A}(8)_{n} \mathrm{Mg}_{2}\left(\mathrm{CF}_{3} \mathrm{SO}_{3}\right)_{2}$

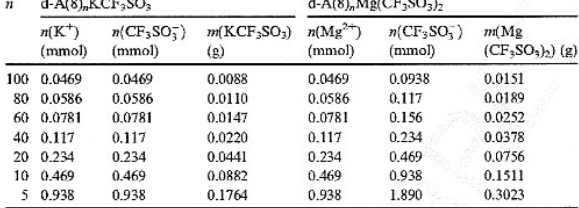

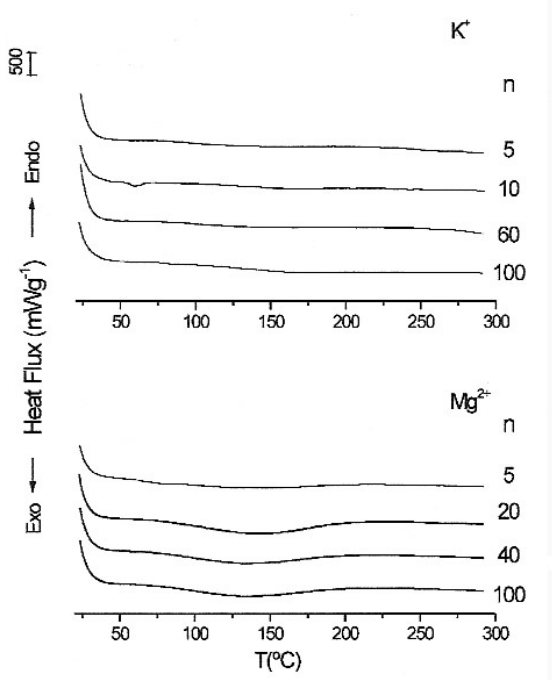

Fig. 1. DSC curves of the d-A $(8)_{n} \mathrm{KCF}_{3} \mathrm{SO}_{3}$ and $\mathrm{d}-\mathrm{A}(8)_{n} \mathrm{Mg}\left(\mathrm{CF}_{3} \mathrm{SO}_{3}\right)_{2}$ di-amidosils.

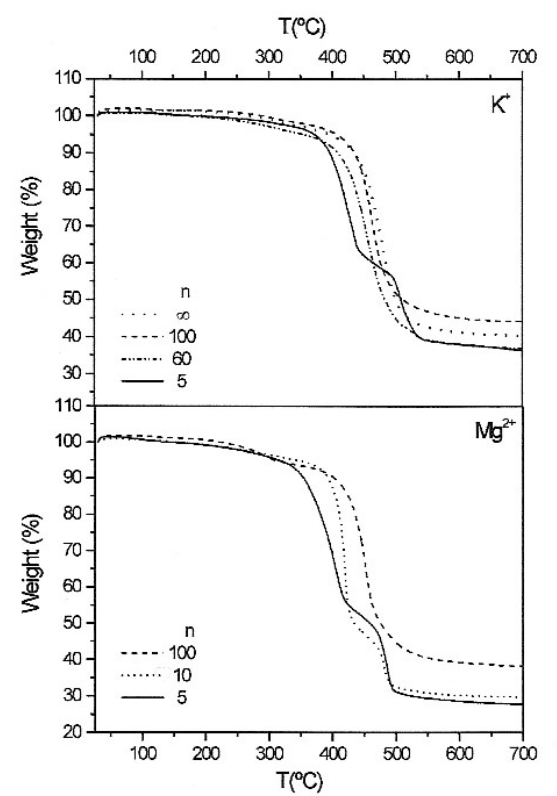

Fig. 2. TGA curves of selected d-A $(8)_{n} \mathrm{KCF}_{3} \mathrm{SO}_{3}$ and d-A $(8)_{n} \mathrm{Mg}\left(\mathrm{CF}_{3} \mathrm{SO}_{3}\right)_{2}$ di-amidosils. 

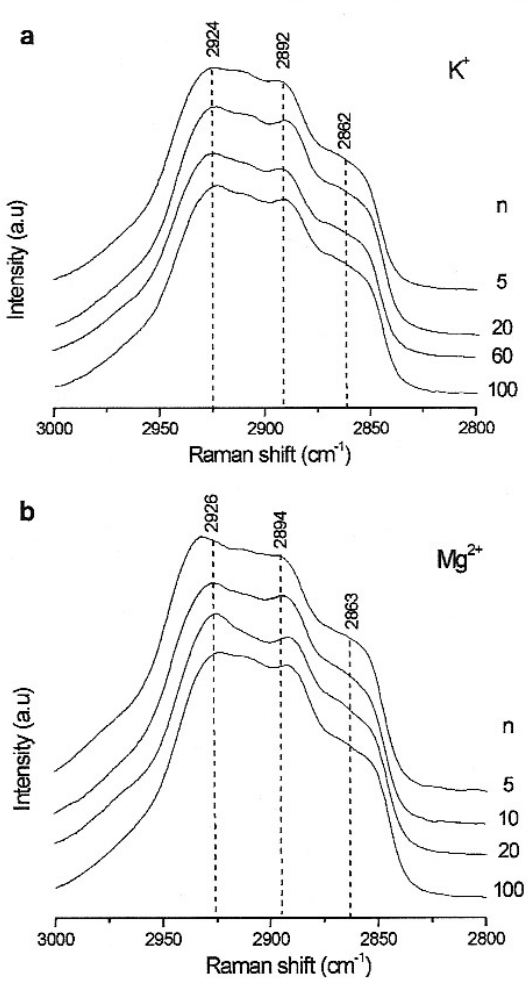

$\mathrm{CH}_{2}$ modes of the $\mathrm{A}(8)_{n} \mathrm{KCF}_{3} \mathrm{SO}_{3}$ (a) and $\mathrm{d}-\mathrm{A}(8)_{1} \mathrm{Mg}\left(\mathrm{CF}_{3} \mathrm{SO}_{3}\right)_{2}$ (b) di-amidosils. The frequencies indicated represent the average value of the frequencies of all the samples considered.
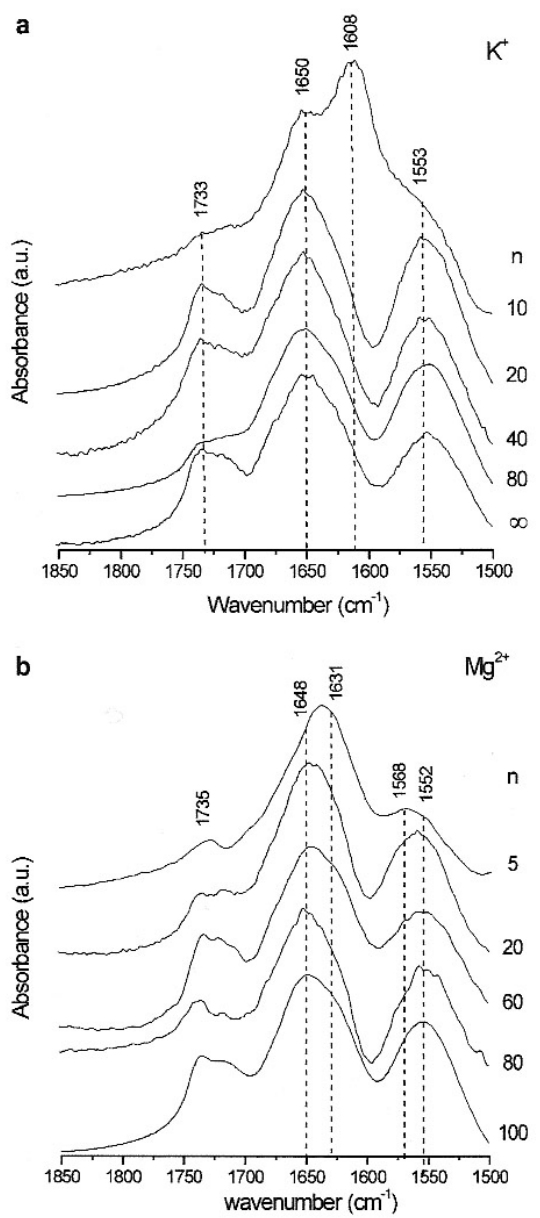

Fig. 4. FT-IR spectra in the Amide $\mathrm{I}$ and Amide II region of the d$\mathrm{A}(8)_{n} \mathrm{KCF}_{3} \mathrm{SO}_{3}$ (a) and $\mathrm{d}-\mathrm{A}(8)_{\pi} \mathrm{Mg}\left(\mathrm{CF}_{3} \mathrm{SO}_{3}\right)_{2}$ (b) di-amidosils. The frequencies indicated represent the average value of the frequencies of all the samples considered. 

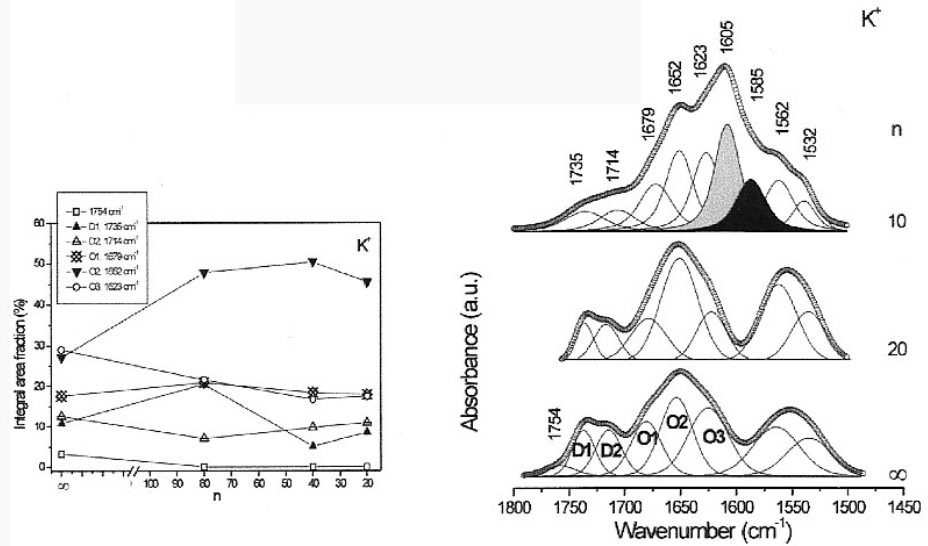

Fig. 5. Curve-fitting results in the $\mathrm{Amide} \mathrm{I}$ and Amide $\mathrm{II}$ of the $\mathrm{d}-\mathrm{A}(8)_{n} \mathrm{KCF}_{3} \mathrm{SO}_{3}$ di-amidosils. The frequencies indicated represent the average value of the frequencies of all the samples considered. Inset: Salt concentration dependence of the integral intensity of the different spectral components resolved in the Amide I region of $\mathrm{d}-\mathrm{A}(8)_{n} \mathrm{KCF}_{3} \mathrm{SO}_{3}$ di-amidosils. The lines drawn are just guides for the eyes.

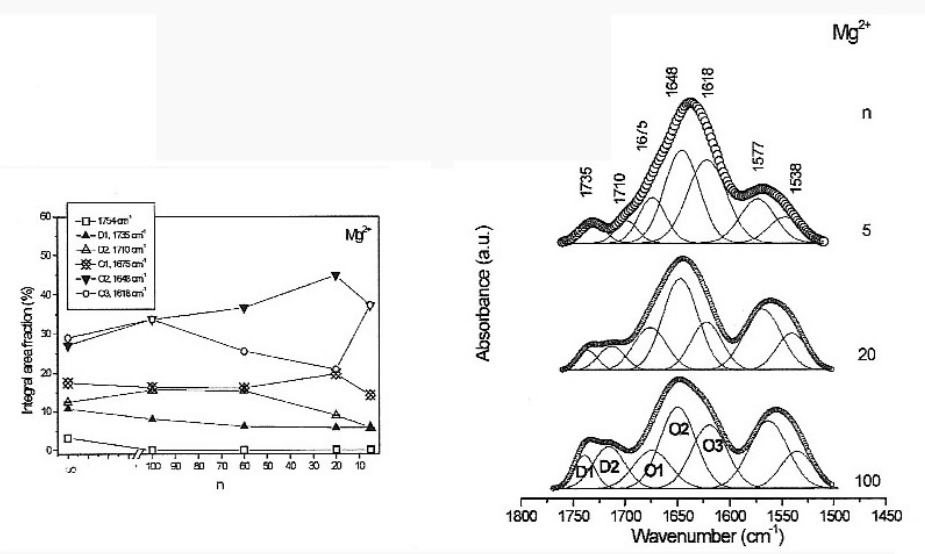

Fig. 6. Curve-fitting results in the Amide $\mathrm{Y}$ and Amide II of the $\mathrm{d}-\mathrm{A}(8)_{n} \mathrm{Mg}\left(\mathrm{CF}_{3} \mathrm{SO}_{3}\right)_{2}$ di-amidosils. The frequencies indicated represent the average value of the frequencies of all the samples considered. Inset: Salt concentration dependence of the integral intensity of the different spectral components resolved in the Amide $\mathrm{I}$ region of $\mathrm{A}(8)_{n} \mathrm{Mg}\left(\mathrm{CF}_{3} \mathrm{SO}_{3}\right)_{2}$ di-amidosils. The lines drawn are just guides for the eyes. 
$\mathbf{a}$
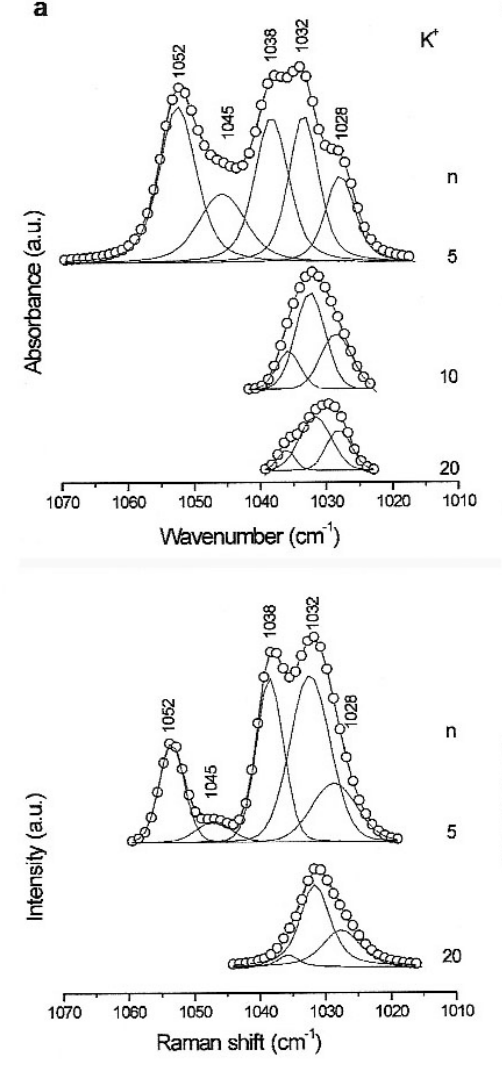

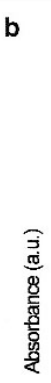
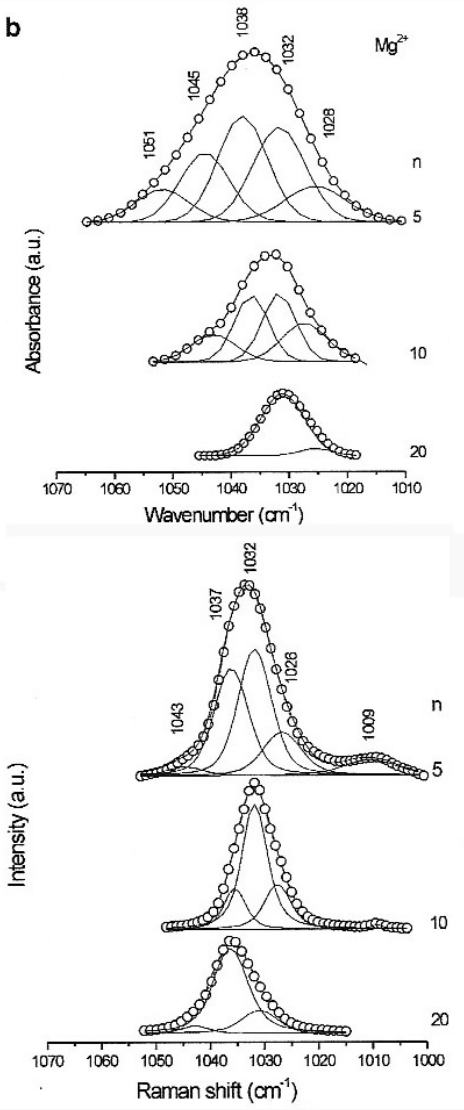

Fig. 7. Curve-fitting of FT-IR (top) and FT-Raman (bottom) $v_{5} \mathrm{SO}_{3}$ region of selected d-A $(8)_{n} \mathrm{KCF}_{3} \mathrm{SO}_{3}$ (a) and d-A $(8)_{n} \mathrm{Mg}\left(\mathrm{CF}_{3} \mathrm{SO}_{3}\right)_{2}$ (b) di-amidosils. The frequencies indicated represent the average value of the frequencies of all the samples considered.
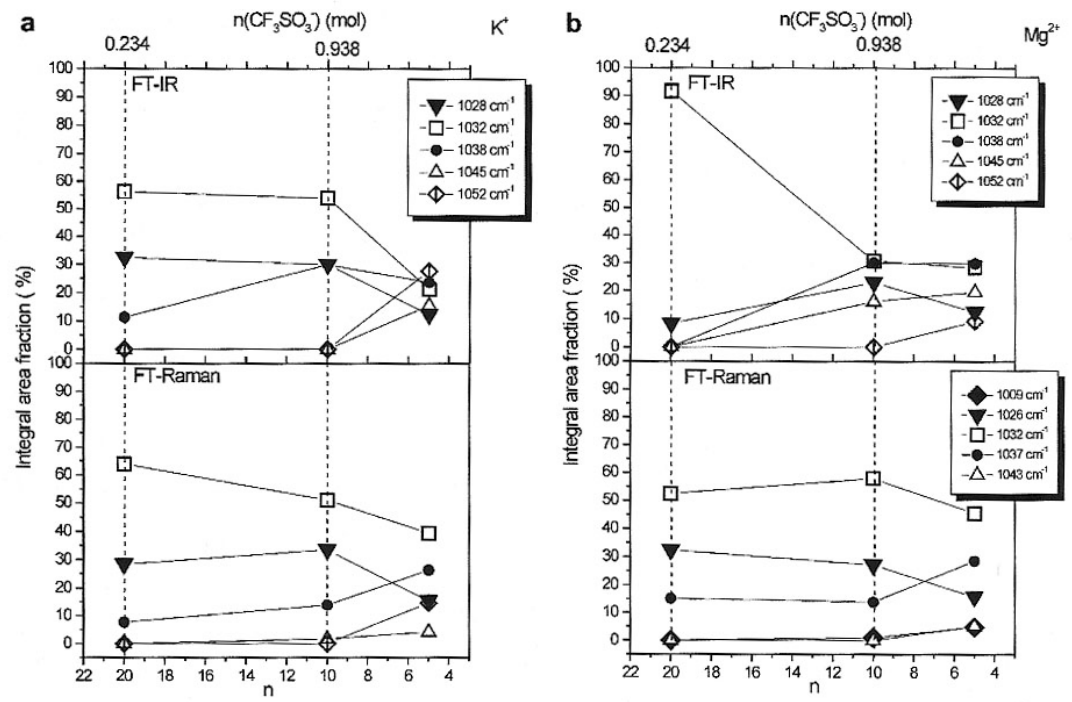

Fig 8. Salt concentration dependence of the integral intensity of the different spectral components resolved in the FT-IR (top) and FT-Raman (bottom) of the d-A(8) ${ }_{n} \mathrm{KCF}_{3} \mathrm{SO}_{3}$ (a) and d-A(8) ${ }_{n} \mathrm{Mg}\left(\mathrm{CF}_{3} \mathrm{SO}_{3}\right)_{2}$ (b) di-amidosils. The lines drawn are just guides for the eyes. 